\title{
Insulin receptor substrates-5 and -6 are poor substrates for the insulin receptor
}

\author{
SOETKIN VERSTEYHE ${ }^{1}$, CHRISTOPHE BLANQUART ${ }^{2,3}$, CORNELIA HAMPE $^{2,3}$, SHAUKAT MAHMOOD ${ }^{1}$, \\ NEVENA CHRISTEFF ${ }^{2,3}$, PIERRE DE MEYTS ${ }^{1}$, STEVEN G. GRAY ${ }^{1,4}$ and TARIK ISSAD ${ }^{2,3}$ \\ ${ }^{1}$ Receptor Systems Biology Laboratory, Hagedorn Research Institute, Novo Nordisk A/S, \\ 2820 Gentofte, Denmark; ${ }^{2}$ Institut Cochin, Université Paris Descartes, CNRS (UMR 8104), 75014 Paris; \\ ${ }^{3}$ Inserm, U567, 75654 Paris, France; ${ }^{4}$ Translational Cancer Research Group, Trinity Centre for Health Sciences, \\ Institute of Molecular Medicine, St James's Hospital, Dublin 8, Ireland
}

Received September 30, 2009; Accepted November 13, 2009

DOI: 10.3892/mmr_00000239

\begin{abstract}
Insulin receptor substrates (IRS)-5 and -6 are two recently identified members of the IRS family. We investigated their roles as insulin receptor substrates and compared them with Src-homology-2-containing (Shc) protein, a well-established substrate. Bioluminescence resonance energy transfer (BRET) experiments showed no interaction between the receptor and IRS-5, while interaction with IRS-6 was not enhanced by insulin. By contrast, Shc showed an insulin-induced BRET response, as did a truncated form of IRS-1 (1-262). While Shc was heavily phosphorylated after stimulation of the insulin receptor, IRS-5 and -6 showed very weak phosphorylation levels. These results suggest that, although these two adaptors have previously been proposed as substrates for the insulin receptor, they are poor substrates for the insulin receptor. This calls into question their relevance to insulin signalling.
\end{abstract}

\section{Introduction}

Insulin is crucial for the regulation of metabolism, growth and development. Binding of insulin to the insulin receptor (IR)

Correspondence to: Dr Soetkin Versteyhe, Institut de Recherche Interdisciplinaire en Biologie Humaine et Moléculaire, Université Libre de Bruxelles, Campus Erasme, Bat. C., CP 602, Route de Lennik 808, 1070 Brussels, Belgium

E-mail: soetkinversteyhe@gmail.com

Abbreviations: BRET, bioluminescence resonance energy transfer; Dok, downstream of kinase; GFP, green fluorescent protein; HEK, human embryonic kidney; IGF, insulin-like grow th factor; IR, insulin receptor; IRS, IR substrate; mBU, milliBRET Unit; PH, pleckstrin homology; PTB, phosphotyrosine binding; Rluc, Renilla luciferase; Shc, Src-homology-2-containing protein; SH2, Src-homology-2; YFP, yellow fluorescent protein

Key words: insulin signalling, insulin receptor substrate protein, bioluminescence resonance energy transfer, phosphorylation leads to the activation of receptor tyrosine kinase and receptor phosphorylation, which enables the binding of docking proteins such as insulin receptor substrates (IRS)-1, -2, -3 and -4 and Src-homology-2-containing protein (Shc). This in turn leads to their phosphorylation and, thereby, intracellular signalling (1).

Until recently, the IRS family included IRS-1, -2, -3 and -4 (2) and three downstream of kinases, Dok-1, -2 and -3 . These seven proteins have similar amino-terminal pleckstrin homology (PH) and similar phosphotyrosine binding (PTB) and carboxyl-terminal phosphorylation domains which, when tyrosine-phosphorylated, dock Src-homology-2 (SH2) domain proteins. Despite their similar domain architecture, the Dok proteins can be distinguished from the IRS family based on sequence homology and functional interactions. Recently, two new members of the family were identified: IRS-5 and -6 (also called Dok-4 and -5, respectively). Based on PH and PTB domain sequence identity, IRS-5 and -6 have been shown to be more closely related to each other than to either the IRS or the Dok family $(3,4)$. Dok-6, defined as a novel member of the Dok-4/5 'subclass' of the Dok family (5), and Dok-7 have also recently been identified (6).

The physiological processes regulated by the Dok family are poorly understood. IRS family members and other receptor tyrosine kinase-associated adaptor molecules are generally involved in signal amplification. Dok-1, -2 and -3 function primarily as inhibitors of tyrosine kinase signalling (7-9). Dok-4 and -5, however, have been shown to be positively involved in tyrosine kinase signalling $(3,10-12)$, although Dok-4 was suggested to be an inhibitor of tyrosine kinase signalling in epithelial cells $(13,14)$. It has been suggested that Dok-4 and -5 are involved in insulin and related insulin-like growth factor (IGF)-I signalling, and play a role as substrates for the insulin and the IGF-I receptor (3). However, contradictory results have been obtained in other studies (13).

Shc is a well-established and -characterized substrate of the IR and plays an important role, mainly in insulin-induced mitogenesis $(15,16)$.

In order to examine the function of IRS-5 and -6 in insulin signalling, we compared the roles of these adapters as IR substrates with the role played by Shc. Contrary to studies 
suggesting that these two proteins are substrates of the IR (3), our results indicate their weakness as IR substrates.

\section{Materials and methods}

Materials. The 4G10 anti-phosphotyrosine antibody was from Upstate Biotechnology (Lake Placid, NY, USA), and the antiGreen Fluorescent Protein (GFP) monoclonal antibody (clones 7.1 and 13.1) was from Roche Applied Science (Indianapolis, IN, USA). All other materials have been described previously $(17,18)$.

Expression vectors. The IR-Renilla luciferase (Rluc) plasmid, coding for the entire IR sequence (19), has been described previously (20).

The yellow fluorescent protein (YFP)-Shc fusion construct was prepared by PCR on pcDNA3 p52Shc (coding for rat p52Shc) with forward primer 5'GGGAAGATCTAACAAGC TGAGTGGAGGCGGCGG3' and reverse primer 5'CCGGAA TTCCTCACACTTTTCGATCCACAGGT3'. After digestion by $\mathrm{Bgl}$ II and EcoR I (restriction sites present in the primers), the insert was cloned into appropriately digested pEYFP-C1 (Clontech, Mountain View, CA, USA).

The IRS-5-YFP and IRS-6-YFP fusion constructs were prepared by PCR on cDNAs (coding for human IRS-5 and -6) using the following primers: IRS-5 forward, 5'CGGAAT TCAAATGGCGACCAATTTCAGTGAC3'; IRS-5 reverse, 5'CCGGGATCCTTCTGGGATGGGGTCTTGGCCTC3'; IRS-6 forward, 5'CTTCGAATTCAAATGGCTTCCAAT TTTAATGACATAG3'; IRS-6 reverse, 5'TACCGTCGACTT GTGCTCAGATCTGTAGGCTGG3'. The PCR products were cloned into pCR2.1 using a TOPO-TA cloning kit (Invitrogen, Denmark). After digestion by EcoR I/BamH I (IRS-5) or EcoR I/Sal I (IRS-6) (restriction sites present in the primers), the insert was shuttled into appropriately digested pEYFP-N1 (Clontech).

The IRS-1(1-262)-YFP fusion construct codes for the first 262 amino acids (and thus for the PH and the PTB domain of the protein) of human IRS-1 coupled to YFP at the C-terminal. The construct was prepared as described by Jacobs et al (21), but with the use of pEYFP-N1 (Clontech) instead of $\mathrm{pEGFP}-\mathrm{N} 2 / 3$.

The constructs were transfected into human embryonic kidney (HEK)-293 cells using FUGENE6 (Roche Diagnostics, Indianapolis, IN, USA) and visualised by fluorescence microscopy.

Bioluminescence resonance energy transfer (BRET) experiments. HEK-293 cells were transfected as described previously (18). To study the interaction between the IR and Shc, 300 ng IR-Rluc cDNA and 300 ng YFP-Shc cDNA or $300 \mathrm{ng}$ empty vector per $35-\mathrm{mm}$ dish were used. For BRET measurements between the IR and IRS-5 or $-6,300 \mathrm{ng}$ IR-Rluc cDNA and $600 \mathrm{ng}$ IRS-5-YFP cDNA, IRS-6-YFP cDNA or empty vector, or $25 \mathrm{ng}$ YFP cDNA and $575 \mathrm{ng}$ empty vector were used. For BRET measurements between the IR and IRS-1(1-262), $300 \mathrm{ng}$ IR-Rluc cDNA and $150 \mathrm{ng}$ IRS-1(1-262)-YFP cDNA or $150 \mathrm{ng}$ empty vector was used. One day after transfection, cells were transferred into 96-well microplates, in which all BRET measurements were carried
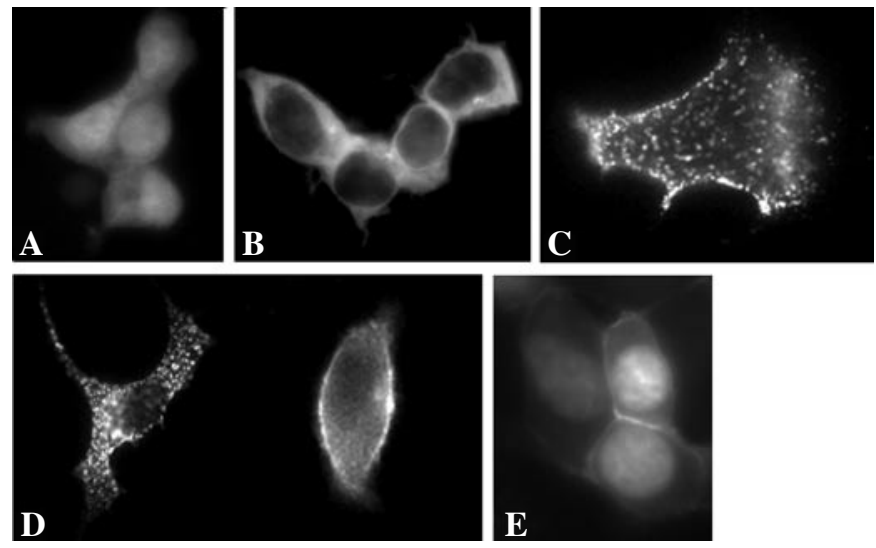

Figure 1. Localization of Shc, IRS-5, IRS-6 and IRS-1(1-262) YFP fusion constructs. HEK-293 cells were transfected with cDNAs encoding YFP alone or YFP-tagged Shc, IRS-5, IRS-6 or IRS-1(1-262). Localization of the proteins was observed using fluorescence microscopy. (A) Expression of YFP alone resulted in a fluorescent signal distributed uniformly throughout the cell. (B) Expression of YFP-Shc resulted in a cytoplasmic distribution of the fluorescence. (C) IRS-5-YFP exhibited a punctuate cytoplasmic fluorescence, while in (D) IRS-6-YFP a fluorescent signal was observed at the plasma membrane and the cytoplasm or in a punctuate cytoplasmic manner. (E) IRS-1(1-262)-YFP exhibited a fluorescent signal partially localized at the plasma membrane.

out on the following day. Results were expressed in milliBRET Units (mBU) as described previously (18).

Phosphorylation measurements. HEK-293 cells were transfected as described previously (18). IR-Rluc cDNA (600 ng) and $300 \mathrm{ng}$ of YFP-Shc, IRS-5-YFP or IRS-6-YFP cDNA or empty vector per $35-\mathrm{mm}$ dish were used. Forty-eight hours after transfection, the cells were incubated with or without $100 \mathrm{nM}$ insulin for $5 \mathrm{~min}$ in Dulbecco's modified Eagle's medium at $37^{\circ} \mathrm{C}$. Proteins were then extracted as previously described (17), subjected to Western blotting (22) and detected using chemiluminescence.

\section{Results}

Localization of Shc, IRS-5, IRS-6 and IRS-1(1-262)-YFP fusion constructs. In HEK-293 cells, YFP-p52Shc localized to the cytoplasmic regions (Fig. 1B). This is in agreement with the findings of Clark et al (23) and Lotti et al (24).

IRS-5-YFP showed a punctuate cytoplasmic localization (Fig. 1C). In fractionation studies, a large quantity of IRS-5-YFP fusion construct was found to be localized in the mitochondria of HEK-293 cells (results not shown). The punctuate cytoplasmic localization was also found by Bedirian et al (13) and Itoh et al (25). Itoh et al also identified these compartments by cell fractionation studies, and confirmed them to be mitochondrial.

In separate cells, but in the same experiment, IRS-6-YFP was either detected at the plasma membrane and in the cytoplasm or showed a punctuate cytoplasmic localization (Fig. 1D). In cell fractionation studies, a small quantity of IRS-6-YFP was found to be present in the mitochondria of HEK-293 cells (results not shown). The localization patterns found were also presented by Shi et al (11). Fu et al (26) also detected IRS-6-YFP at the plasma membrane. 
A

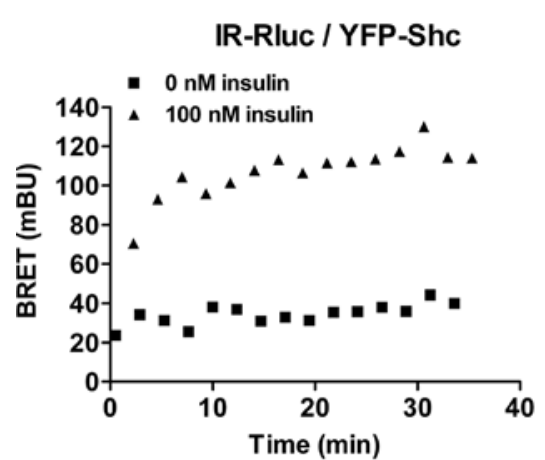

B

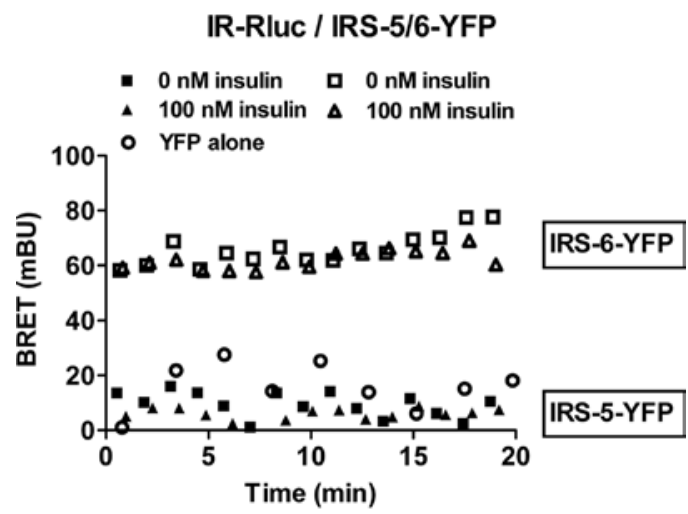

C

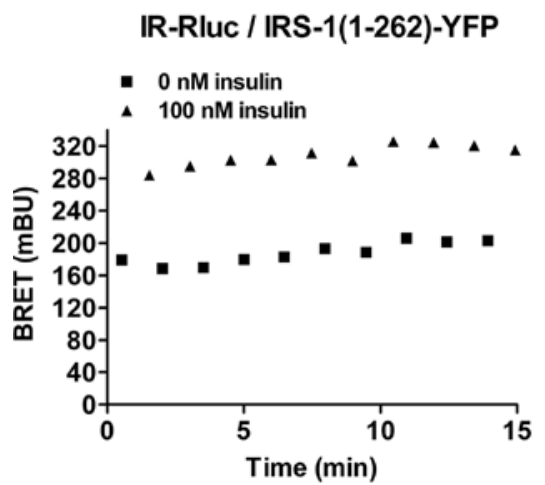

Figure 2. Real-time interaction of the insulin receptor with Shc, IRS-5, IRS-6 and IRS-1(1-262). HEK-293 cells were transfected with IR-Rluc + YFP-Shc, IR-Rluc + IRS-5-YFP, IR-Rluc + IRS-6-YFP, IR-Rluc + YFP or IR-Rluc + IRS-1(1-262)-YFP. The BRET signal was measured in real-time in living cells after stimulation with $0(\boldsymbol{\square}, \square)$ or $100 \mathrm{nM}(\boldsymbol{\Lambda}, \triangle)$ insulin. (A) Dynamics of the interaction between IR-Rluc and YFP-Shc before and after stimulation. Results are representative of three independent experiments. (B) Dynamics of the interaction between IR-Rluc and IRS-5-YFP or IRS-6-YFP before and after stimulation. In order to indicate the specificity of the interaction between IR and IRS-6, the BRET signal after transfection with IR-Rluc and YFP alone at a similar YFP/Rluc ratio is shown (O). $\mathbf{m}$ and $\mathbf{\Delta}$, IRS-5; $\square$ and $\Delta$, IRS-6. Results are representative of two independent experiments. (C) Dynamics of the interaction between IR-Rluc and IRS-1(1-262)-YFP before and after stimulation. Results are representative of two independent experiments.

IRS-1(1-262)-YFP localized partially to the plasma membrane (Fig. 1E). This localization pattern is in accordance with that presented by Jacobs et al (21) for IRS-1(1-262)-GFP.

These results show that the fusion constructs used in this study are correctly expressed according to the currently available knowledge.
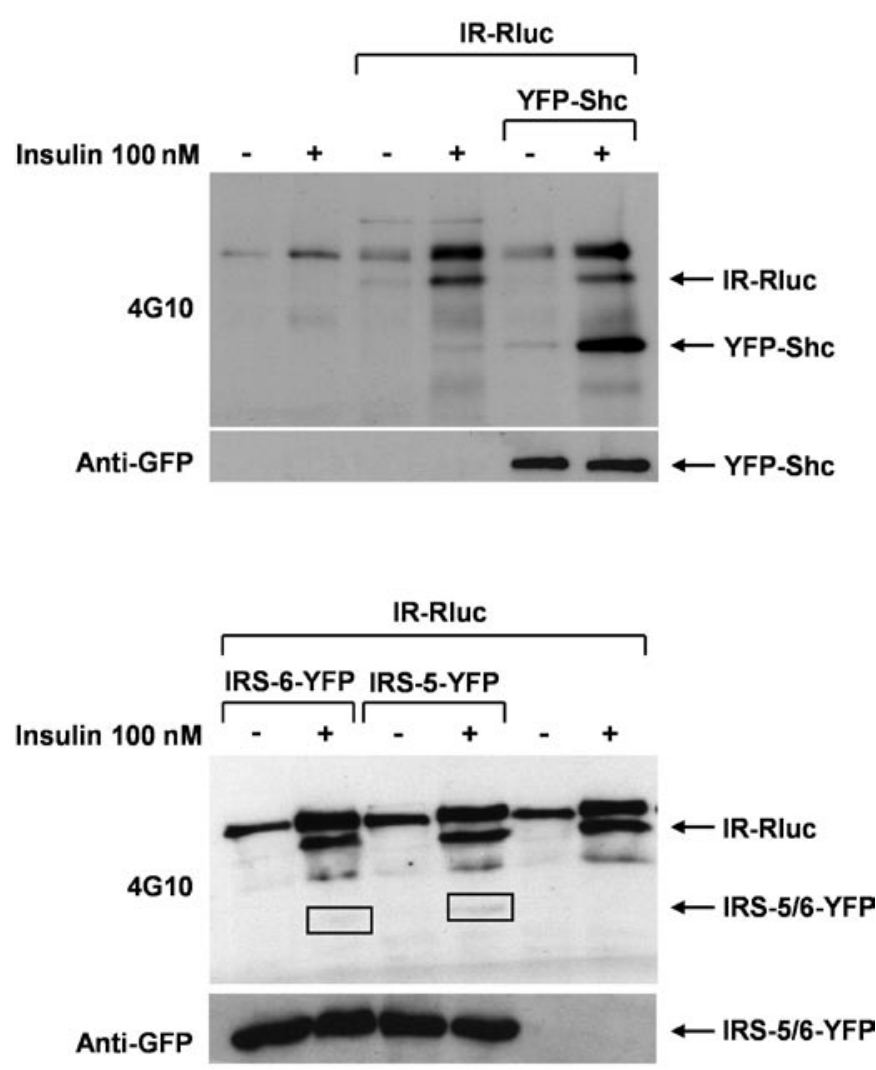

Figure 3. Phosphorylation of Shc, IRS-5 and IRS-6 in response to insulin. HEK-293 cells were transfected with empty vector, IR-Rluc, IR-Rluc + YFP-Shc, IR-Rluc + IRS-5-YFP or IR-Rluc + IRS-6-YFP. Forty-eight hours after transfection, the cells were incubated in the absence or presence of $100 \mathrm{nM}$ insulin for $5 \mathrm{~min}$. Proteins were extracted and tyrosine phosphorylation was determined by immunoblotting using an anti-phosphotyrosine antibody (4G10). Results are representative of two independent experiments.

Real-time interaction of the insulin receptor with Shc, IRS-5, IRS- 6 and IRS-1(1-262). Real time interactions between the IR and Shc, IRS-5, IRS-6 and, as a positive control, IRS-1(1-262) were measured in living cells by BRET. HEK-293 cells were transfected with IR-Rluc and YFP-Shc, IRS-5-YFP, IRS-6-YFP or IRS-1(1-262)-YFP. In the BRET assay, Rluc is excited by the addition of its substrate, coelenterazine. If the potential interaction partners bring Rluc and YFP $<100 \AA$ apart, an energy transfer occurs between luciferase and YFP, and a signal emitted by YFP can be detected.

Shc interacted with the IR in an insulin-dependent manner (Fig. 2A). In cells transfected with IRS-5, virtually no BRET signal could be detected (Fig. 2B). With IRS-6, a substantial and specific basal BRET signal was detected, but insulin had no effect on this signal (Fig. 2B). As a positive control within the IRS family, the BRET signal observed between IR-Rluc and IRS-1(1-262)-YFP was enhanced by insulin stimulation (Fig. 2C).

Phosphorylation of Shc, IRS-5 and IRS-6 in response to insulin. We decided to evaluate whether the insulin-induced BRET response between the IR and Shc, IRS-5 and IRS-6 correlated with their phosphorylation levels after the stimulation of the cells with insulin. Insulin strongly induced the tyrosine phosphorylation of Shc (Fig. 3). Shc was substantially 
phosphorylated in comparison to the phosphorylation levels of the IR. In contrast, insulin induced only a very poor phosphorylation of IRS-5 and -6 (Fig. 3). In the same experiment, the IR itself was heavily phosphorylated after stimulation.

\section{Discussion}

The physiological roles of IRS-5 (Dok-4) and -6 (Dok-5), two recently discovered members of the IRS/Dok family of adaptor proteins, are still very poorly understood. We compared the roles of these two adaptors as substrates of the IR with Shc, a well-established and -characterized insulin receptor substrate.

By BRET measurements, Shc was demonstrated to interact with the IR in an insulin-dependent manner, while IRS-5 did not show any BRET response. IRS- 6 produced a solid and specific basal BRET response; however, this signal was not enhanced by stimulation with insulin. By contrast, a truncated form of the major insulin receptor substrate IRS-1 (amino acids 1-262) did, just like Shc, show a solid basal BRET signal that was enhanced by stimulation with insulin (a truncated form of IRS-1 was used since full length IRS-1-YFP shows an anomalous cell localization) (21). Just like IRS-1(1-262), IRS-5 and -6 were fused to YFP at their C-terminal, leaving the N-terminal PH domain and the PTB domain free for interaction with the cell membrane and the receptor. However, the lack of BRET response between IRS-5 and the IR does not completely rule out an interaction between these two proteins. Since the energy transfer (leading to a BRET signal) between Rluc and YFP depends not only on the distance but also on the relative orientation of the two partners, the lack of BRET response with IRS-5 could be due to an unfavourable orientation of the Rluc-YFP BRET pair for energy transfer to occur. The difference in basal BRET levels between IRS-5 and -6 might also be explained by their different cellular localizations. IRS-5 was shown in the present and previous studies (25) to be localized in the mitochondria. This could mean that the protein is not as readily available for a BRET interaction. IRS-6 partially localized to the cell membrane and the cytoplasm, possibly providing the opportunity for an easier interaction and a higher basal BRET.

The lack of an insulin-inducible BRET signal for IRS-6 could indicate that IRS-6 is constitutively bound to the IR and does not get recruited to the receptor after stimulation. Although it is unclear whether IRS-6 binds to the same locus on the IR as its two major substrates, IRS-1 and -2, our data suggest that it may act as a competitive inhibitor of IRS-1 and -2 . This warrants further investigation, including whether the interaction of IRS- 6 and the IR can be regulated by factors other than insulin.

In addition, we showed that, while Shc phosphorylation is heavily induced by insulin stimulation, both IRS-5 and -6 show very weak phosphorylation levels after IR activation in HEK-293 cells. To be categorized as genuine 'IRS', an adaptor must be tyrosine-phosphorylated by the IR in addition to having the appropriate PH-PTB domain architecture. Our data therefore suggest the weakness of IRS-5 and -6 as insulin receptor substrates. In contrast with our results, Cai et al found IRS-5 and -6 to be phosphorylated after stimulation of the insulin and the related IGF-I receptor in HEK-293 and Chinese hamster ovary cells (3). However, Bedirian et al showed that, in COS cells, overexpressed IGF-I receptor was able to phosphorylate itself, but unable to phosphorylate IRS-5, even after stimulation with IGF-I (13). This group was also unable to detect IRS-5 phosphorylation in epithelial cells or in IRS-5-overexpressing 293 cells after treatment with IGF-I.

Although these contradictory results are possibly due to differences in experimental conditions, it is clear that further studies are needed to elucidate which role these adaptors play as substrates for the insulin and IGF-I receptor and in insulin and IGF-I receptor signalling. Animal knockouts of these adaptors would provide valuable information and further clarify the physiological roles of IRS-5 and -6.

\section{Acknowledgements}

Soetkin Versteyhe was the recipient of an Industrial $\mathrm{PhD}$ scholarship from the Danish Ministry of Science, Technology and Innovation. Steven Gray was the recipient of a BIO+IT postdoctoral fellowship from the Oeresund IT Academy. This study was supported by the Association pour la Recherche sur le Cancer (Grant no. 3781) and by the Ligue contre le Cancer, Comite de Paris (Grant no. R04/75/75). The Hagedorn Research Institute and the Receptor Systems Biology Laboratory are independent basic research components of Novo Nordisk A/S.

\section{References}

1. Taniguchi CM, Emanuelli B and Kahn CR: Critical nodes in signalling pathways: insights into insulin action. Nat Rev Mol Cell Biol 7: 85-96, 2006.

2. Giovannone B, Scaldaferri ML, Federici M, Porzio O, Lauro D, Fusco A, Sbraccia P, Borboni P, Lauro R and Sesti G: Insulin receptor substrate (IRS) transduction system: distinct and overlapping signaling potential. Diabetes Metab Res Rev 16: 434-441, 2000.

3. Cai D, Dhe-Paganon S, Melendez PA, Lee J and Shoelson SE: Two new substrates in insulin signaling, IRS5/DOK4 and IRS6/ DOK5. J Biol Chem 278: 25323-25330, 2003.

4. Favre C, Gérard A, Clauzier E, Pontarotti P, Olive D and Nunès JA: DOK4 and DOK5: new Dok-related genes expressed in human T cells. Genes and Immunity 4: 40-45, 2003.

5. Crowder RJ, Enomoto H, Yang M, Johnson EM and Milbrandt J: Dok-6, a Novel p62 Dok family member, promotes Ret-mediated neurite outgrowth. J Biol Chem 279: 42072-42081, 2004.

6. Okada K, Inoue A, Okada M, et al: The muscle protein Dok-7 is essential for neuromuscular synaptogenesis. Science 312: 1802-1805, 2006.

7. Lemay S, Davidson D, Latour S and Veillette A: Dok-3, a novel adapter molecule involved in the negative regulation of immunoreceptor signaling. Mol Cell Biol 20: 2743-2754, 2000.

8. Di Cristofano A, Niki M, Zhao M, Karnell FG, Clarkson B, Pear WS, Van Aelst L and Pandolfi PP: p62(dok), a negative regulator of Ras and mitogen-activated protein kinase (MAPK) activity, opposes leukemogenesis by p210(bcr-abl). J Exp Med 194: 275-284, 2001.

9. Van Slyke P, Coll ML, Master Z, Kim H, Filmus J and Dumont DJ: Dok-R mediates attenuation of epidermal growth factor-dependent mitogen-activated protein kinase and Akt activation through processive recruitment of c-Src and Csk. Mol Cell Biol 25: 3831-3841, 2005.

10. Grimm J, Sachs M, Britsch S, Di Cesare S, Schwarz-Romond T, Alitalo K and Birchmeier W: Novel p62dok family members, dok-4 and dok-5, are substrates of the c-Ret receptor tyrosine kinase and mediate neuronal differentiation. J Cell Biol 154: 345-354, 2001.

11. Shi L, Yue J, You Y, Yin B, Gong Y, Xu C, Qiang B, Yuan J, Liu Y and Peng X: Dok5 is substrate of TrkB and TrkC receptors and involved in neurotrophin induced MAPK activation. Cell Signal 18: 1995-2003, 2006. 
12. Uchida M, Enomoto A, Fukuda T, et al: Dok-4 regulates GDNFdependent neurite outgrowth through downstream activation of Rap1 and mitogen-activated protein kinase. J Cell Sci 119: 3067-3077, 2006.

13. Bedirian A, Baldwin C, Abe J, Takano T and Lemay S: Pleckstrin homology and phosphotyrosine-binding domain-dependent membrane association and tyrosine phosphorylation of Dok-4 an inhibitory adapter molecule expressed in epithelial cells. J Biol Chem 279: 19335-19349, 2004.

14. Baldwin $\mathrm{C}$, Bedirian $\mathrm{A}, \mathrm{Li} \mathrm{H}$, Takano $\mathrm{T}$ and Lemay $\mathrm{S}$ Identification of Dok-4b, a Dok-4 splice variant with enhanced inhibitory properties. Biochem Biophys Res Commun 354: 783-788, 2007.

15. Sasaoka T and Kobayashi M: The functional significance of Shc in insulin signaling as a substrate of the insulin receptor. Endocr J 47 373-381, 2000.

16. Ravichandran KS: Signaling via She family adapter proteins. Oncogene 20: 6322-6330, 2001.

17. Boute N, Pernet K and Issad T: Monitoring the activation state of the insulin receptor using bioluminescence resonance energy transfer. Mol Pharmacol 60: 640-645, 2001.

18. Boute N, Boubekeur S, Lacasa D and Issad T: Dynamics of the interaction between the insulin receptor and protein tyrosinephosphatase 1B in living cells. EMBO Rep 4: 313-319, 2003.

19. Ullrich A, Bell JR, Chen EY, et al: Human insulin receptor and its relationship to the tyrosine kinase family of oncogenes. Nature 313: 756-761, 1985.

20. Blanquart C, Gonzalez-Yanes $\mathrm{C}$ and Issad T: Monitoring the activation state of insulin/insulin-like growth factor-1 hybrid receptors using bioluminescence resonance energy transfer. Mol Pharmacol 70: 1802-1811, 2006.
21. Jacobs AR, LeRoith D and Taylor SI: Insulin receptor substrate-1 pleckstrin homology and phosphotyrosine-binding domains are both involved in plasma membrane targeting. J Biol Chem 276: 40795-40802, 2001

22. Issad T, Combettes M and Ferre P: Isoproterenol inhibits insulinstimulated tyrosine phosphorylation of the insulin receptor without increasing its serine/threonine phosphorylation. Eur J Biochem 234: 108-115, 1995.

23. Clark SF, Martin S, Carozzi AJ, Hill MM and James DE: Intracellular localization of phosphatidylinositide 3-kinase and insulin receptor substrate-1 in adipocytes: potential involvement of a membrane skeleton. J Cell Biol 140: 1211-1225, 1998.

24. Lotti LV, Lanfrancone L, Migliaccio E, Zompetta C, Pelicci G, Salcini AE, Falini B, Pelicci PG and Torrisi MR: Sch proteins are localized on endoplasmic reticulum membranes and are redistributed after tyrosine kinase receptor activation. Mol Cell Biol 16: 1946-1954, 1996.

25. Itoh S, Lemay S, Osawa M, Che W, Duan Y, Tompkins A, Brookes PS, Sheu SS and Abe J: Mitochondrial Dok-4 recruits Src kinase and regulates NF-kappaB activation in endothelial cells. J Biol Chem 280: 26383-26396, 2005.

26. Fu G, Zhang F, Cao L, Xu ZZ, Chen YZ, Wang GY and He C: Constitutive plasma membrane targeting and microdomain localization of Dok5 studied by single-molecule microscopy. Biophys Chem 136: 13-18, 2008. 